\title{
Dispersion Monitoring for High-Speed WDM Networks via Two-Photon Absorption in a Semiconductor Microcavity
}

\author{
P.J. Maguire, K. Bondarczuk, L.P. Barry, J. O’Dowd*, W.H. Guo* \\ M. Lynch*, A.L. Bradley*, J.F. Donegan*, H. Folliot** \\ Research Institute for Networks and Communications Engineering, Dublin City University, Dublin 9, Ireland \\ *Semiconductor Optronics Group, University of Dublin ,Trinity College, Dublin 2, Ireland \\ **Laboratoire de Physique des Solides, INSA, Rennes, France \\ Tel: (3531)700 5884,Fax: (3531)700 5508,e-mail:maguirep@eeng.dcu.ie
}

\begin{abstract}
Due to the continued demand for bandwidth, network operators have to increase the data rates at which individual wavelengths operate at. As these data rates will exceed $100 \mathrm{Gbit} / \mathrm{s}$ in the next $5-10$ years, it will be crucial to be able to monitor and compensate for the amount of chromatic dispersion encountered by individual wavelength channels. This paper will focus on the use of the novel nonlinear optical-to-electrical conversion process of Two-Photon Absorption (TPA) for dispersion monitoring. By incorporating a specially designed semiconductor microcavity, the TPA response becomes wavelength dependent, thus allowing simultaneous channel selection and monitoring without the need for external wavelength filtering.

Keywords: dispersion monitoring, wavelength division multiplexing, two-photon absorption, microcavity.

\section{INTRODUCTION}

Due to the continued growth of the Internet and the introduction of new broadband services such as video-ondemand and mobile telephony, there is a need to increase the data rates of individual channels in a WavelengthDivision Multiplexing (WDM) system in order to meet demand. One of the major limiting factors to the maximum transmission distance of high-bit rate signals is chromatic dispersion (CD). As the amount of CD encountered fluctuates due to dynamic network configurations and variations in environmental conditions such as temperature, it is essential to continuously monitor the amount of $\mathrm{CD}$ of individual wavelength channels. One technique to monitor $\mathrm{CD}$ is to employ a peak-power dependent detector, which has the ability to differentiate between two optical pulses with the same average power, but differing pulse durations. This would allow the CD of individual channels to be monitored. An ideal candidate for this peak-power dependent detector is the nonlinear optical-to-electrical conversion process of Two-Photon Absorption (TPA) in semiconductors.
\end{abstract}

\section{TWO-PHOTON ABSORPTION}

Two-Photon Absorption (TPA) is a nonlinear optical-to-electrical conversion process that occurs in semiconductors when two photons are absorbed to generate a single electron-hole pairing. It occurs when a photon of energy $E_{p h}$ is incident on the active region of a semiconductor device with a band gap energy exceeding $E_{p h}$ but less than $2 E_{p h}$. Under these conditions, individual photons do not possess sufficient energy to produce an electron-hole pair. However an electron-hole pair can be produced by the simultaneous absorption of two photons, were the summation of the individual photon energies is greater than the band gap energy. The resulting photocurrent produced is proportional to the square of the incident optical power falling on the detector [1]. It is this nonlinear response, combined with TPA's ultra-fast response time $\left(10^{-14} \mathrm{~s}\right.$ at $1550 \mathrm{~nm}$ [2]), that enables TPA to be considered for use in high-speed optical signal processing applications such as the monitoring of $\mathrm{CD}$ of individual wavelength channels.

One of the major problems associated with the use of TPA for optical signal processing applications is its inherent inefficiency, requiring either high optical intensities typically not found in an optical communications network, or a long interaction length for response enhancement [1]. One way in which the efficiency of the TPA process can be greatly enhanced is to place the active region within a semiconductor microcavity structure [3]. The microcavity works by placing mirrors at either end of the active region of the semiconductor, resulting in the formation of very strong optical fields within the cavity. This can be viewed as an increase in the interaction length of the active region. This leads to a reduction of the device length when compared with waveguide structures, as well as a significant enhancement of the TPA generated photocurrent by four orders of magnitude when compared to non-cavity devices [3]. In addition, the microcavity benefits from having a wavelength selective response, making such devices suitable for WDM and hybrid Wavelength Division Multiplexed/Optical Time Division Multiplexed (hybrid WDM/OTDM) applications as the selection and optical signal processing functionality (optical sampling, switching, dispersion monitoring) can be carried out

This work is supported under Enterprise Ireland's Advanced Technology Research Programme (ATRP/2002/301a/b) and Science Foundation Ireland's CSET Centre for Telecommunications Value Driven Research (03/IE3/I405), and Investigator Programme (03/IN3/I427). 
simultaneously using a single device without the need for external filtering. In addition, by tilting the device, it is possible to vary the peak of the wavelength resonance of the microcavity. This angle tuning alters the selected wavelength channel allowing multiple wavelengths to be monitored using a single device, therefore removing the need for expensive re-growth of different detectors for different WDM channels [4].

\subsection{Device Performance}

Prior to carrying out any experimental work, some initial characterization of the TPA microcavity samples was carried out. Figure $1 a$ displays a plot of the photocurrent generated as a function of the incident peak optical power close to the resonance wavelength peak. It shows a square dependence of the photocurrent generated on the incident optical intensity, which is evidence of the TPA process, with residual SPA occurring at low energies. However, there is over 3 orders of magnitude of nonlinear response with the current device. Figure $1 b$ shows a plot of the photocurrent generated as a function of incident optical wavelength. As can be seen, the cavity response is dependent on the incident wavelength, with a cavity wavelength resonance of $1556 \mathrm{~nm}$, and a measured spectral linewidth of $4.2 \mathrm{~nm}$. Figure $1 b$ also shows that the peak wavelength resonance is 3 -orders of magnitude greater when compared to the photocurrent generated for off-resonance wavelengths for the current device.
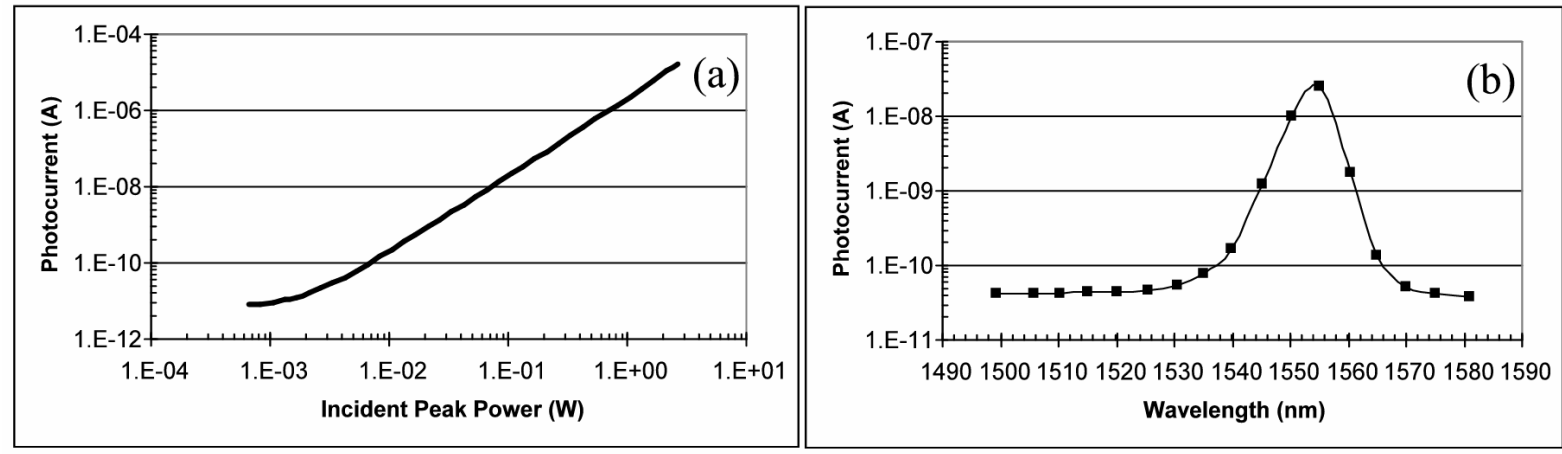

Figure 1. (a) Plot of the photocurrent as a function of incident optical peak power at the cavity wavelength resonance; (b) Plot of the photocurrent generated as a function of incident optical wavelength.

\section{TPA-BASED DISPERSION MONITORING}

Assuming that the incident optical pulses have a Gaussian shape, the average TPA photocurrent generated is given by [5]:

$$
i_{t p a, a v e}=e V \frac{\beta T}{2 \sqrt{\pi} h v \tau}\left(I_{a v e}\right)^{2}
$$

where $V$ is the volume of the active region of the TPA detector, $\beta$ is the TPA coefficient, $T, \tau$ and $I_{a v e}$ are the period, pulse width and average intensity of the incident optical signal. Thus equation (1) states that as TPA is a peak power dependent process, two optical pulses with equal energies but different pulse widths will generate different photocurrents. Therefore the TPA photocurrent generated is inversely proportional to the pulse width, and it is this fact that allows TPA to be used for dispersion monitoring.

Our proposed TPA-based dispersion monitor is shown in Figure $2 a$. The incident WDM signal $\left(\lambda_{1}, \lambda_{2}, \ldots . \lambda_{N}\right)$ enter a 10:90 passive optical fibre coupler. 10\% of the WDM signal is incident on the TPA microcavity which is mounted on a rotation stage, with the remaining signal allowed to continue to propagate across the network. The wavelength channel that coincides with the resonance wavelength of the microcavity will be detected, will all other channels rejected as their wavelengths reside outside the enhancement bandwidth of the cavity. As TPA is a peak-power dependent process, it can differentiate between optical pulses with the same average optical powers, but differing pulse widths, corresponding to pulses that have been temporally broadened due to CD. By simply varying the angle of the microcavity $\theta$, different wavelength channels can be monitored, with a larger angle of incidence shortening the resonant wavelength of the cavity [6]. Thus the TPA effect in the microcavity leads to an angle-dependent response of the incident WDM signal. It has already been shown that pulse characterisation can be performed over a wide wavelength tuning range $(>50 \mathrm{~nm})$ with a single TPA microcavity mounted on a rotational stage by exploiting this angular response [6]. Such a wide tuning range would allow a single device to be used to monitor dispersion in a number of different wavelength channels in C-band right through to the L-band, without the need for growth of different devices. Also, as the width of the resonant response can be altered by changing the refractive index difference between the materials comprising the mirrors placed at either end of the active region, a microcavity can be designed to meet the channel spacing of the target 
WDM network. Figure $2 b$ shows a conceptual diagram of how the dispersion monitor could be incorporated into a dispersion equaliser for a high-speed WDM system.
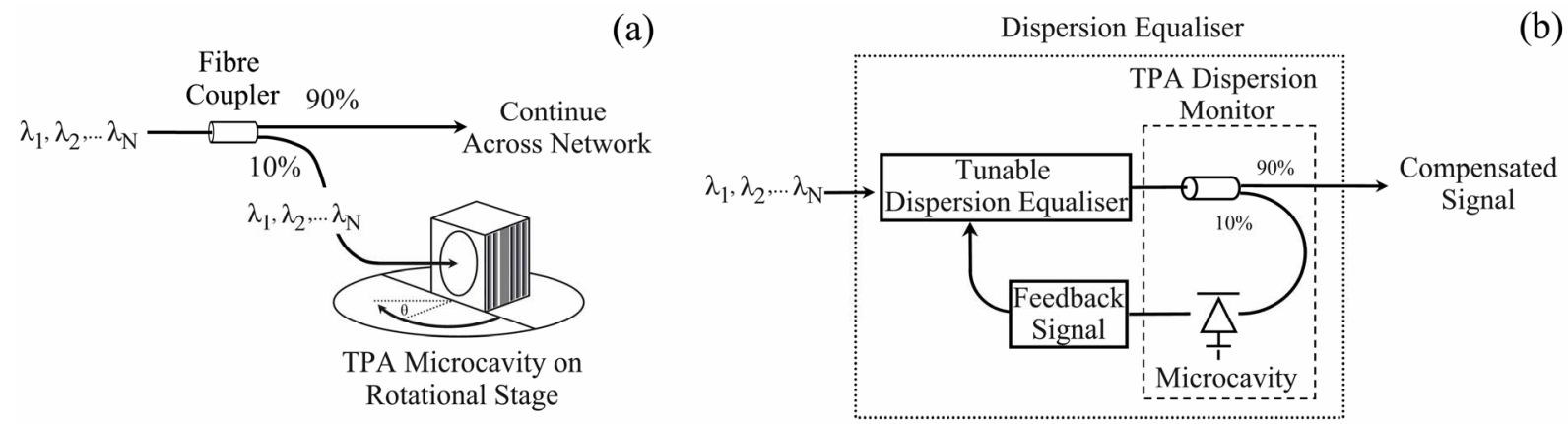

Figure 2. (a) Configuration of proposed TPA-based: (a) dispersion monitor; (b) dispersion equaliser.

The CD-dependent signal from the TPA microcavity is fed back to feedback circuitry which would drive a tunable dispersion equaliser, allowing dispersion of the incident signal to be altered, optimising system performance. The tunable dispersion equaliser could be a nonlinear chirped fibre Bragg grating similar to that reported in [7].

\section{EXPERIMENTAL VERIFICATION}

According to Equation 1, two pulses with equal energies but different pulse widths (and hence different peak intensities) should generate different TPA photocurrents. In order to verify this, and demonstrate the suitability of utilising TPA for dispersion monitoring, the experimental setup shown in Fig. 3 was employed.

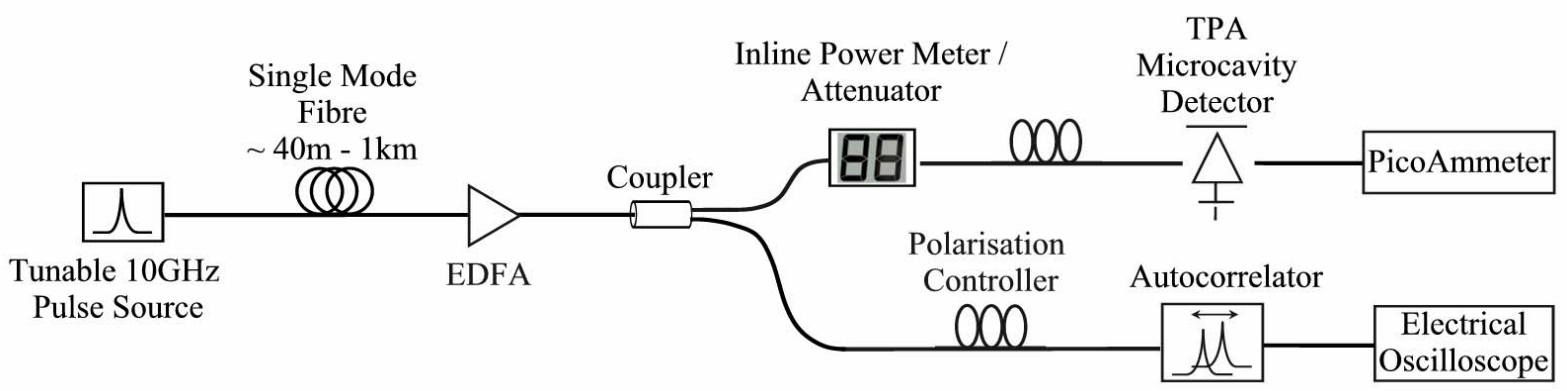

Figure 3. Experimental setup used for TPA-based dispersion monitoring.

The main concept is to increase the optical pulse width by propagating an optical pulse train through various lengths of single mode fibre (SMF-28) ranging from $40 \mathrm{~m}$ to $1 \mathrm{~km}$. The TPA photocurrent generated is then recorded as a function of dispersed pulse. The optical pulse train was provided by a $10 \mathrm{GHz}$ pulse source, which generates optical pulses with durations $\sim 2$ ps over a tuneable wavelength range from 1480 to $1580 \mathrm{~nm}$. The optical pulses were then propagated through various lengths of single mode fibre mode $(D=16 \mathrm{ps} / \mathrm{km} \cdot \mathrm{nm}$ at $1550 \mathrm{~nm}$ ) and amplified by a low-noise EDFA before entering a $3 \mathrm{~dB}$ optical coupler. One of the coupler outputs enters an inline power meter / attenuator and a polarization controller before being incident on the TPA microcavity. The inline power meter / attenuator allowed the average optical intensity of the signal falling on the TPA microcavity to be continuous monitored and maintained during the course of the experiment. The photocurrent generated by the microcavity was then measured using a picoammeter. The second output of the coupler entered a polarization controller followed by a SHG-based autocorrelator which allowed for the measurement of the optical pulse durations after fibre propagation.

\section{EXPERIMENTAL RESULTS}

Figure $4 a$ shows a plot of the TPA photocurrent generated by the microcavity as a function of optical pulse duration for two different wavelengths, the microcavity resonance wavelength of $1556 \mathrm{~nm}$, and an off-resonance wavelength of $1545 \mathrm{~nm}$. The average optical intensity, as measured on the inline power meter before the TPA microcavity, was kept constant at approximately $10 \mathrm{dBm}$ during the course of the experiment. When the incident wavelength was set to $1556 \mathrm{~nm}$, the TPA photocurrent dropped of at $1 / \tau$ as the pulse width broadened after propagation in longer lengths of fibre (as expected from Equation 1). 

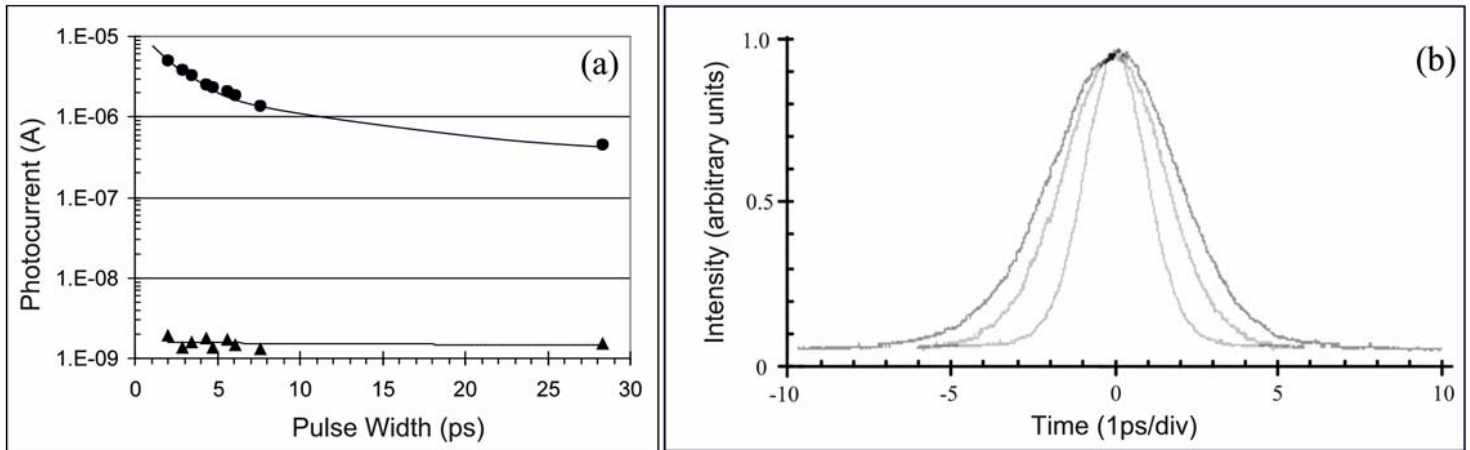

Figure 4. (a) Plot of the pulse width dependence of TPA photocurrent at microcavity resonance wavelength of $1556 \mathrm{~nm}(\boldsymbol{\bullet})$ and off-resonance wavelength of $1545 \mathrm{~nm}(\mathbf{\Delta})$; (b) Autocorrelation traces after $100 \mathrm{~m}, 200 \mathrm{~m}$ and $300 \mathrm{~m}$ of $S F M-28$.

Also shown in Fig. $4 a$ is the TPA response for an off-resonant wavelength (1545 nm). The response is no longer dependent on the peak optical intensity as the wavelength is outside the enhancement bandwidth of the microcavity, with the response no longer dependent on the optical pulse width. The photocurrent generated at $1545 \mathrm{~nm}$ wavelength was approximately 2-orders less than at $1556 \mathrm{~nm}$, which is consistent with microcavity wavelength response shown in Fig. $1 b$. Figure $4 b$ shows the autocorrelation trace after pulse propagation through $100 \mathrm{~m}, 200 \mathrm{~m}$ and $300 \mathrm{~m}$ of SMF respectively. As is clearly shown, with the pulse width increased from $\sim 3$ ps to 5 ps from $100 \mathrm{~m}$ to $300 \mathrm{~m}$ of fibre propagation.

\section{CONCLUSIONS}

In order to optimize the performance of future high-speed WDM networks, it will be essentially to continuously monitor the amount of chromatic dispersion experienced by each individual wavelength channel. This paper has demonstrated that dispersion monitoring can be carried out using a TPA microcavity, as the photocurrent generated by the device is inversely proportional to the pulse width. As the microcavity has a wavelengthselective response, and by incorporating angle tuning, it is possible to monitor multiple wavelengths using a single device. This removes the need for expensive re-growth of different detectors for different WDM channels. Such a dispersion monitor could be incorporated into a dispersion equalizer for a high-speed WDM network.

\section{REFERENCES}

[1] H. Folliot, M. Lynch, A.L. Bradley, T. Krug, L.A. Dunbar, J. Hegarty, J.F. Donegan, L.P. Barry, "Twophoton-induced photoconductivity enhancement in semiconductor microcavities, "A theoretical investigation,” J. Opt. Soc. Amer. B. Opt. Phys., vol. 19, pp. 2396-2402, Oct. 2002.

[2] J. Donegan, "Two-photon absorption speeds optical switching," Lightw. Europe, vol. 1, pp. 31 , July, 2002.

[3] H. Folliot, M. Lynch, A.L. Bradley, L.A. Dunbar, J. Hegarty, J.F. Donegan, L.P. Barry, J.S. Roberts, G. Hill, "Two-photon absorption photocurrent enhancement in bulk AlGaAs semiconductor microcavities," Appl. Phys. Lett, vol. 80, pp. 1328-1330, Feb. 2002.

[4] K. Naganuma, K. Mogi, H. Yamada, "General Method for Ultrashort Light Pulse Chirp Measurement," IEEE J. Quantum Electron, vol. 25, pp. 1225-1233, Jun. 1989.

[5] Z. Zheng, A.M. Weiner, J.H. Marsh, M.M. Karkhanehchi, "Ultrafast optical thresholding based on twophoton absorption GaAs Waveguide Photodetectors,” IEEE Photon. Technol. Lett, vol. 9, pp. 493-495, Apr. 1997.

[6] T. Krug, W.H.Guo, J. O'Dowd, M. Lynch, A.L. Bradley, J.F. Donegan, P.J. Maguire, L.P. Barry, H. Folliot, "Resonance Tuning of Two-Photon Absorption Microcavities for Wavelength-Selective Pulse Monitoring," IEEE Photon. Technol. Lett, vol. 18, pp. 433-435, Jan. 2006.

[7] T. Inui, T. Komukai, K. Mori, T. Morioka: “160-Gb/s Adaptive Dispersion Equalisation Using an Asynchronous Dispersion-Induced Chirp Monitor,” J. Lightwave Technol., vol. 23, pp. 2039-2045, Jun. 2005. 